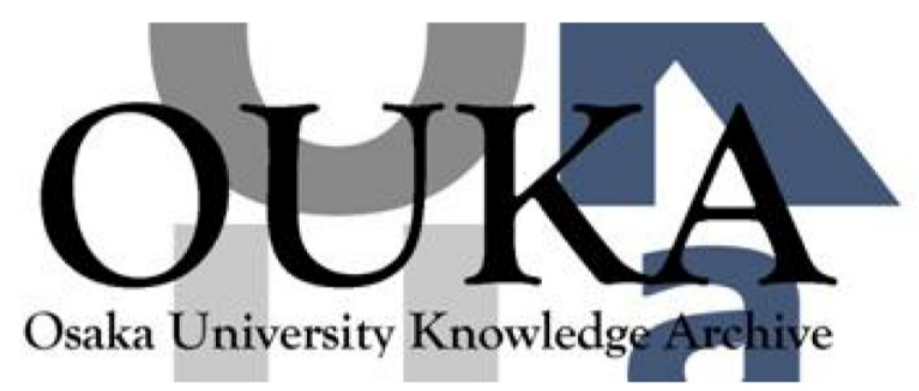

\begin{tabular}{|c|l|}
\hline Title & $\begin{array}{l}\text { In Situ HREM Observation of Crystalline-to-Gas } \\
\text { Transition in Nanometer-Sized Ag Particles }\end{array}$ \\
\hline Author(s) & Lee, J.-G.; Lee, J.; Tanaka, T. et al. \\
\hline Citation & Physical Review Letters. 96(7) p. 075504 \\
\hline Issue Date & $2006-02-23$ \\
\hline oaire:version VoR \\
\hline URL & https://hdl. handle. net/11094/25980 \\
\hline rights & 02006 The American Physical Society \\
\hline Note & \\
\hline
\end{tabular}

Osaka University Knowledge Archive : OUKA

https://ir. Library. osaka-u. ac. jp/

Osaka University 


\title{
In Situ HREM Observation of Crystalline-to-Gas Transition in Nanometer-Sized Ag Particles
}

\author{
J.-G. Lee, ${ }^{1, *}$ J. Lee, ${ }^{2}$ T. Tanaka, ${ }^{3}$ and H. Mori ${ }^{1}$ \\ ${ }^{1}$ Research Center for Ultra-High Voltage Electron Microscopy, Osaka University, Japan \\ ${ }^{2}$ Division of Materials Science and Engineering, Korea University, Korea \\ ${ }^{3}$ Department of Materials Science and Processing, Osaka University, Japan
} (Received 16 April 2005; published 23 February 2006)

\begin{abstract}
Sublimation of silver atoms from a nanometer-sized silver particle, namely, the crystalline-to-gas transition, has been directly observed by high resolution electron microscopy. Remarkable sublimation of silver atoms took place at $950 \mathrm{~K}$ from an approximately 9-nm-sized crystalline silver particle on the graphite substrate. The sublimation continued until the whole silver particle disappeared. No melting of the particle occurred during this process. The height-to-width ratio of a nanometer-sized silver particle on graphite at $950 \mathrm{~K}$ was about 0.65 , and the ratio did not change so much during the sublimation-induced shrinkage of the particle.
\end{abstract}

Interest in nanometer-sized particles has grown remarkably for the last decades and a lot of work has been done to understand the origin of the unique structures and properties of these particles [1,2]. With respect to the structure, recent developments in high resolution electron microscopy (HREM) greatly contributed to the understanding of the phase stability of nanometer-sized particles of not only pure substances [3,4] but also alloys [5-10]. Most of these HREM works, however, have been concerned with the direct confirmation of such phase transitions as the solidto-liquid transition [6] and the liquid-to-glass transition [5,7], and to the authors' best knowledge, no HREM observations have been reported on the solid-to-gas transition (i.e., sublimation). It is of significance to directly confirm whether a nanometer-sized particle on a substrate would undergo a solid-to-gas transition prior to the whole particle disappearing or whether a particle would first undergo a solid-to-liquid (i.e., melting) transition and then undergo a liquid-to-gas transition until all the constituent atoms of the particle evaporate. It is also of interest to examine the change of morphology of a particle during such a solidto-gas (or liquid-to-gas) transition as a function of particle size. This is because the particle morphology reflects not only the magnitude of the interaction between the constituent atom of the particle and the supporting substrate but also the magnitude of the surface tension [11]. Therefore, analysis of the particle morphology as a function of particle size would provide valuable information on the size dependence of the particle-substrate interaction and of the surface tension.

Based upon these premises, a series of in situ HREM experiments to study the solid-to-gas transition and the morphology change of nanometer-sized particles has been carried out. The present Letter presents results of such experiments. Specifically, it describes the behavior of a nanometer-sized silver particle supported on the graphite substrate as revealed by in situ HREM. Silver particles on graphite were chosen as the vehicle of the study because the carbon solubility in silver can be ignored even at the melting point of silver, which makes the discussion simple [12]. Nanometer-sized particles were produced and observed in the same vacuum chamber of HREM without exposing particles to any undesired atmosphere because it is essential to keep the surface of particles as clean as possible during the experiments. This is, of course, because these particles possess an extremely high surface-to-volume ratio.

Nanometer-sized silver particles were prepared on the graphite substrate using a side-entry holder which was equipped with an evaporator at its tip, in a Hitachi HF2000 type $200 \mathrm{kV}$ HREM. A schematic illustration of the holder can be seen in a previous paper [13]. The evaporator consisted of two spiral-shaped tungsten filaments. One of the filaments was attached to a flake of graphite which served as a supporting substrate and the other with silver as a source material. Prior to experiment, the flake was baked at around $1100 \mathrm{~K}$ for $60 \mathrm{~s}$ to obtain a cleaned surface. After the graphite substrate was cooled down to around $600 \mathrm{~K}$, silver was then evaporated from the second filament onto the substrate kept at the same temperature, and nanometersized silver particles were produced on the edge of the graphite flake. The substrate temperature was so chosen that the size and density of silver particles on the graphite substrate was appropriate for in situ experiments mentioned below. After being formed on the graphite substrate, silver particles were once cooled down to room temperature. The particles were then subjected to in situ annealing experiments in the microscope. Namely, the particles were heated quite slowly up to $950 \mathrm{~K}$ and kept at the temperature, and any phase transition and morphology change which may occur in a nanometer-sized silver particle during the annealing at $950 \mathrm{~K}$ were monitored in situ using a supersensitive CCD camera (AMT, XR-60BFE) with a frame rate of 9 frames/s. Images obtained were recorded on videotapes. All micrographs presented in this Letter were reproduced from the videotapes. The base pressure 
in the specimen chamber of this microscope was below $7 \times$ $10^{-7} \mathrm{~Pa}$.

A typical crystalline-to-gas transition in a nanometersized silver particle on the graphite substrate kept at $950 \mathrm{~K}$ is depicted in Fig. 1. The number inserted in each micrograph indicates relative time in seconds. The $0.23_{6} \mathrm{~nm}$-spaced fringes seen in the particle correspond to the (111) lattice fringes of pure silver and the $0.33_{5} \mathrm{~nm}$-spaced fringes correspond to the (0001) lattice fringes of graphite substrate. It is seen that the crystalline silver particle is oriented with the (111) face parallel to the (0001) face of graphite substrate. This orientation relationship is consistent with that reported in the literature [14]. As shown in Figs. 1(a)-1(f), it is obvious that evaporation of silver atoms from an approximately 9-nm-sized silver particle occurred at $950 \mathrm{~K}$, which eventually led to the complete disappearance of the silver particle [Fig. 1(f)]. Here it should be noted that the evaporation of silver atoms
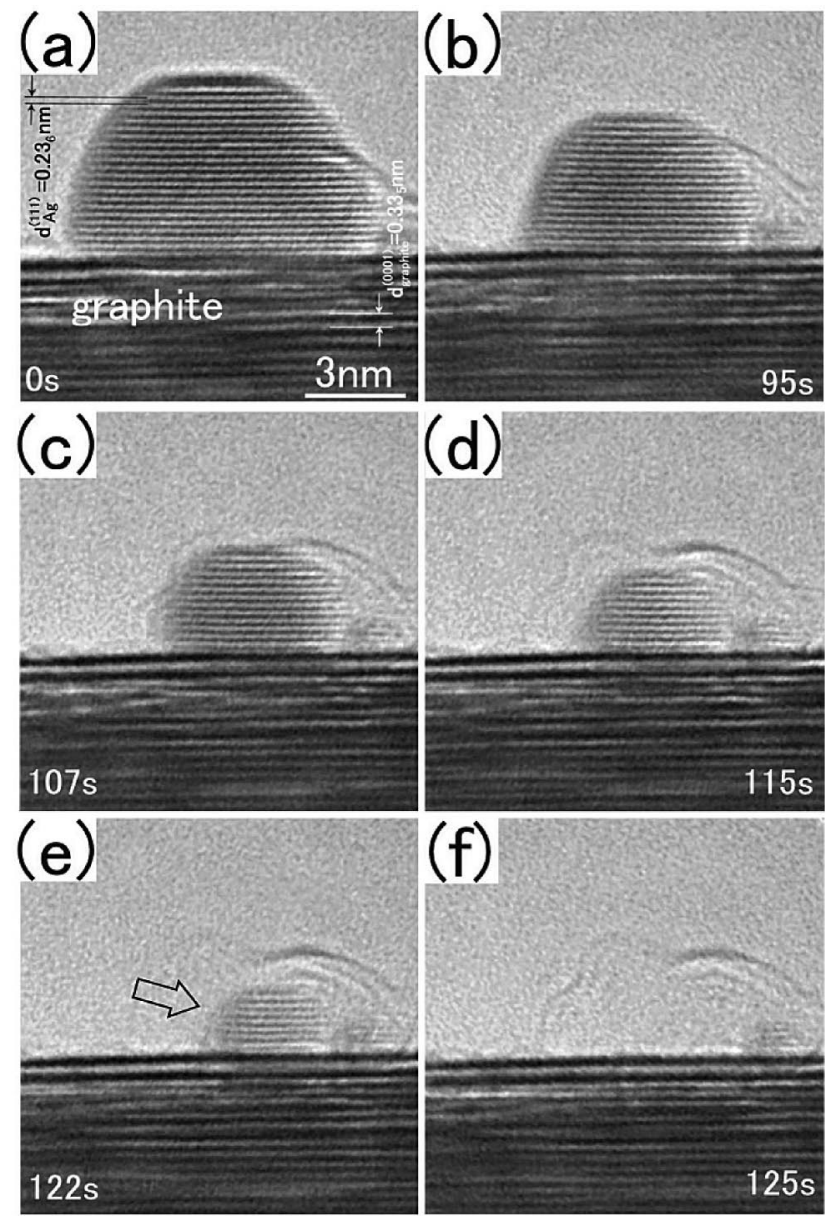

FIG. 1. A typical crystalline-to-gas transition in a nanometersized silver particle on the graphite substrate kept at $950 \mathrm{~K}$. The number inserted in each micrograph indicate relative times in seconds. Remarkable sublimation of silver atoms occurred from a crystalline silver particle at $950 \mathrm{~K}$ under the vacuum of $7 \times 10^{-7} \mathrm{~Pa}$ (a)-(e), and it continued until whole the crystalline silver particle disappeared (f). During this process, no melting of the silver particle occurred. occurred from a crystalline silver particle (that is, sublimation occurred), and it continued until whole the crystalline silver particle disappeared [Fig. 1(f)]. In fact, in the particle shown in Fig. 1(e), eight lattice fringes (arrow) were observed, indicating the particle is not in a liquid state but in a crystalline state. It is emphasized that no crystalline-to-liquid transition took place during this process.

A similar phenomenon (i.e., sublimation) of silver was reported by Sambles et al. 34 years ago [15]. They conducted a conventional, not a high resolution, electron microscopy study of evaporating small silver particles supported on an amorphous carbon substrate. Based upon the careful analysis of the particle-size reduction, they reported that the evaporation of silver atoms from small solid silver particles occurred without melting. On the other hand, in the present work, by using atomic resolution electron microscopy it was directly confirmed that a silver particle on the graphite substrate exhibited sublimationinduced shrinkage and no crystalline-to-liquid transition took place prior to the complete disappearance of the particle. The present observation is consistent with the result by Sambles et al.

It is well known that the melting point decreases with decreasing size of particles [16-18]. For example, the melting point depression in nanometer-sized silver particles was reported by Castro and Reifenberger [19]. They measured the field-emission current from individual particles deposited on the end surface of a tungsten field emitter and found that the temperature at which the field-emission current exhibited an abrupt change depended on the particle size. They took such a temperature as the melting point. By this technique, the melting point of silver particle was reported to decrease down to below $950 \mathrm{~K}$ when the size of particles becomes smaller than $9 \mathrm{~nm}$ in diameter [19]. However, this result by Castro and Reifenberger is inconsistent with the present experimental result that a 3-nmsized crystalline silver particle was stable against melting even at $950 \mathrm{~K}$ [Fig. 1(e)] and it continued shrinking by sublimation without melting until it disappeared completely [see Fig. 1(f)]. From this comparison, it seems safe to consider that the linear reciprocal relationship between the melting point and the particle size which is widely accepted as a universal relationship between the melting point and the particle size [20] cannot hold, at least not in nanometer-sized silver particles supported on the graphite substrate. This fact suggests that there still remain unknown factors which affect the size dependence of melting point established by an accumulation of theoretical and experimental results when the particle size decreases down to several nanometers in diameter. To obtain an in-depth understanding on the origin of the size dependence of melting point, further work is needed.

Next, the morphology of silver particles on the graphite substrate was studied as a function of particle size. As a quantitative measure of the particle morphology, the height-to-width ratio (for definition, see the inset in 


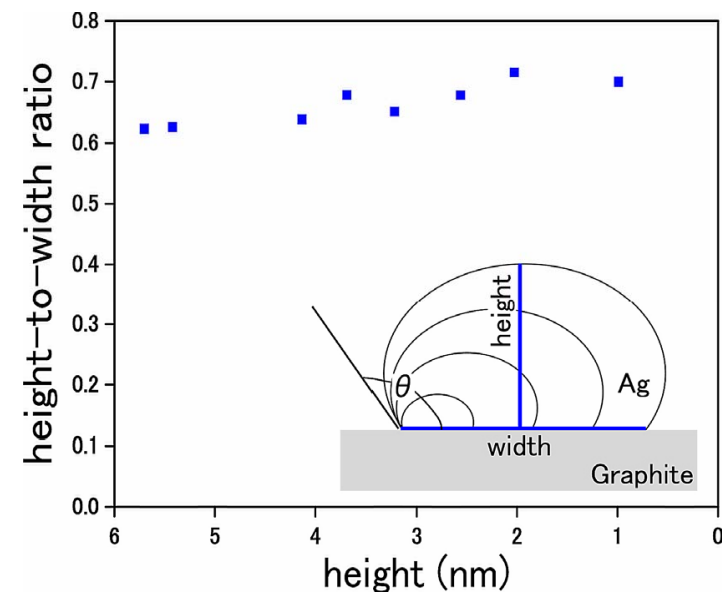

FIG. 2 (color online). Size dependence of the height-to-width ratio of a silver particle on graphite at $950 \mathrm{~K}$. The height-towidth ratio was almost constant, 0.65 (or the ratio seems to even slightly increase), during the sublimation-induced shrinkage of the silver particle.

Fig. 2) was taken [21], since it is known that this ratio is sensitively influenced by the magnitude of the particlesubstrate interaction and by the value of the surface tension [11]. For example, the height-to-width ratio would increase with decreasing magnitude of the particle-substrate interaction and/or with increasing value of the surface tension [11]. Based upon these points, the ratio was continuously measured during the sublimation-induced shrinkage of the silver particle. A typical result of the size dependence of the height-to-width ratio is shown in Fig. 2. The data have been taken from the silver particle on the graphite substrate kept at $950 \mathrm{~K}$ depicted in Fig. 1. In Fig. 2, the abscissa corresponds to the height of the particle, and the height decreased during the sublimation of silver (from the left to the right on the abscissa). As seen from Fig. 2, the heightto-width ratio is almost constant, 0.65 , during the sublimation-induced shrinkage of the particle. From this result, it seems safe to conclude that both the surface tension and the interaction of silver with the carbon substrate do not change so much in the particle-size (height) range below $9 \mathrm{~nm}$. This would provide a piece of useful information on the particle-size dependence of wettability, which is one of current topics in the research field of surface and interface in the size-constrained systems [2123].

The present work can be summarized as follows: (1) An atomic resolution electron microscopy study has been carried out to examine successive stages of sublimationinduced disappearance of $\mathrm{Ag}$ particle. It is revealed that no crystalline-to-liquid transition took place in a silver particle prior to the complete disappearance. (2) It is also revealed that the height-to-width ratio of a silver particle, which reflects the wettability between the Ag particle and the substrate, remained almost constant during the shrinkage of the particle.

This work was supported by "Priority Assistance of the Formation of Worldwide Renowned Centers of Research-The 21st Century COE Program (Project: Center of Excellence for Advanced Structural and Functional Materials Design)" from the Ministry of Education, Sports, Culture, Science and Technology of Japan. A part of the present work was supported by the Ministry of Education, Sports, Culture, Science and Technology of Japan under Grant-in-Aids for Scientific Research (Grants No. 15074213 and No. 17206064).

*Corresponding author. Electronic address: jg-lee@uhvem.osaka-u.ac.jp

[1] W. P. Halperin, Rev. Mod. Phys. 58, 533 (1986).

[2] R. P. Andres et al., J. Mater. Res. 4, 704 (1989).

[3] S. Iijima and T. Ichihashi, Phys. Rev. Lett. 56, 616 (1986).

[4] P. M. Ajayan and L. D. Marks, Phys. Rev. Lett. 63, 279 (1989).

[5] H. Mori and H. Yasuda, Scr. Mater. 44, 1987 (2001).

[6] J.-G. Lee, H. Mori, and H. Yasuda, Phys. Rev. B 65, 132106 (2002).

[7] J.-G. Lee, H. Mori, and H. Yasuda, Phys. Rev. B 66, 012105 (2002).

[8] J.-G. Lee and H. Mori, Phys. Rev. B 70, 144105 (2004).

[9] J.-G. Lee and H. Mori, Philos. Mag. 84, 2675 (2004).

[10] J.-G. Lee and H. Mori, Phys. Rev. Lett. 93, 235501 (2004).

[11] A. W. Adamson, Physical Chemistry of Surfaces (John Wiley \& Sons, New York, 1990).

[12] T. B. Massalski et al., Binary Alloy Phase Diagrams (American Society for Metals, Metals Park, OH, 1986).

[13] J.-G. Lee and H. Mori, J. Vac. Sci. Technol. A 21, 32 (2003).

[14] O. Dezellus and N. Eustathopoulos, Scr. Mater. 40, 1283 (1999).

[15] J. R. Sambles, L. M. Skinner, and N. D. Lisgarten, Proc. R. Soc. A 318, 507 (1970).

[16] J. R. Sambles, Proc. R. Soc. A 324, 339 (1971).

[17] Ph. Buffat and J.-P. Borel, Phys. Rev. A 13, 2287 (1976).

[18] G. L. Allen, R. A. Bayles, W. W. Gile, and W. A. Jesser, Thin Solid Films 144, 297 (1986).

[19] T. Castro and R. Reifenberger, Phys. Rev. B 42, 8548 (1990).

[20] M. Zhang et al., Phys. Rev. B 62, 10548 (2000).

[21] J. Lee, K. Morita, and T. Tanaka, Mater. Trans., JIM 44, 2659 (2003).

[22] A. Checco, P. Guenoun, and J. Daillant, Phys. Rev. Lett. 91, 186101 (2003).

[23] D. Quere, Nat. Mater. 3, 79 (2004). 